\title{
COMPARISON BETWEEN EQUINE AND RABBIT ANTI-THYMOCYTE GLOBULIN (ATG) USED IN CONDITIONING FOR PEDIATRIC PATIENTS UNDERGOING HEMATOPOIETIC STEM CELL TRANSPLANTATION
}

\author{
Sunil Bhat ${ }^{1}$, Ruchi Chaudhary ${ }^{2}$, Sohini Chakraborty ${ }^{2}$, Shobha Badiger ${ }^{2}$, and Pooja Mallya ${ }^{2}$ \\ ${ }^{1}$ RESIDENCE \\ ${ }^{2}$ Narayana Hrudayalaya Health City Bangalore
}

July 31, 2020

\begin{abstract}
Background: HSCT related complications and disease relapse are major obstacles in successful transplantation. Addition of ATG has shown to reduce rejection and Graft Vs Host Disease (GVHD) incidence significantly. Procedure: Here we report single center retrospective analysis for relative efficacy of rabbit and equine ATG during conditioning of pediatric patients who underwent HSCT. Results: Rabbit or Equine ATG were used as a part of conditioning regimen in 117 pediatric patients. Equine ATG group was labeled as group-1 and rabbit ATG as group-2. Both groups were analyzed for transplant related complications and outcome. In thalassemia, outcomes were comparable except Group-1 had more cases of proven bacterial infection $(\mathrm{p}=0.07)$ and veno-occlusive disease $(\mathrm{p}=0.03)$. In SAA, Group-1 had early neutrophil and platelet engraftment with less number of proven bacterial infections. Acute GVHD, chronic GVHD, median chimerism at day+30 and day+90 and overall survival (OS) was comparable in two groups, both in thalassemia and SAA patients. Analysis of 7 hematological malignancy cases and 10 immunodeficiency disorders also showed similar results. Conclusion: In conclusion, effectiveness of rabbit and horse ATG were similar in thalassemia, primary immunodeficiency patients and hematological malignancy while in SAA equine ATG use was associated with early neutrophil engraftment but OS in both groups were comparable.
\end{abstract}

\section{Introduction}

Hematopoietic stem cell transplant (HSCT) is a curative option for many hematological and nonhematological disorders. Multiple strategies have been evolved over a period of time for reduction of GVHD, transplant rejection and other complications.

Anti-thymocyte globulin (ATG) is the purified IgG fraction of sera from rabbits or horses that are immunized with human thymocytes or $\mathrm{T}$ cell lines. Several multicenter, prospective, randomized, or nonrandomized studies evaluated the efficacy of ATG against GVHD. ${ }^{1-4}$ Addition of antithymocyte globulin (ATG) to pretransplantation conditioning has been shown to decrease the incidence of acute graft-versus-host disease (aGVHD) and chronic graft-versus-host disease (cGVHD) in recipients of both unrelated and related donor grafts ${ }^{5-17}$.

Both equine and rabbit ATG are available in India. Rabbit ATG is available with brand name Thymoglobulin (manufactured by Sanofi) and equine ATG as Thymogam and Atgam (manufactured by Bharat serum \& Pfizer respectively). No study has compared role of different varieties of ATG in pre stem cell transplant conditioning. 
In our center both rabbit and horse ATG were used in pre-transplant conditioning hence a retrospective analysis was done to look for relative efficacy in form of various transplant associated infectious and noninfectious complications and survival.

\section{Methods}

This was a retrospective analysis from hospital records of 117 pediatric patients who underwent stem cell transplantation and received anti-thymocyte globulin as a part of conditioning regimen in our center from January 2013 to May 2017. Equine ATG group received $90 \mathrm{mg}$ per $\mathrm{kg}$ of ATG over 3 to 4 days and was labelled as Group-1, while rabbit ATG group received $7.5 \mathrm{mg}$ per $\mathrm{kg}$ of ATG and was labelled as Group-2. Both groups were compared for different transplant associated complications, rejection, and overall survival.

Standard selection criteria for selecting donors and standard definitions for engraftment, GVHD, OS and even free survival (EFS) were employed ${ }^{3}$. Post-transplant chimerism was done by RT-PCR routinely at day $+28,3$ months, 6 months and then at 1 year.

Statistical analysis:

SPSS 20.0 software was used to analyze data and the survival curve was made by the Kaplan-Meier method. Survival differences between groups were estimated by the log-rank test. Patient, disease, and transplantrelated characteristics were compared using the chi-square test. $\mathrm{P}$ value of [?] .05 was considered significant.

\section{Results}

Data from 117 patients including 79 thalassemia, 21 patients of aplastic anemia, 7 patients of acute leukemia and 10 patients of immunodeficiency disorders were analyzed, and results were as shown below:

\section{Thalassemia:}

Out of total 79 thalassemic patients, 47 patients were in group-1 while 32 patents in group-2. Baseline characteristics of two groups are shown in table 1. Group-1 had a greater number of class III thalassemia and median age was also higher in group-1. Busulfan based conditioning was used in class I and II thalassemia while thiotepa-trisula based conditioning was used in class III. Transplant characteristics including stem cells source, stem cell dose and type of transplant used were comparable in both groups.

Engraftment:

Out of 79 patients, 77 achieved neutrophil engraftment and 71 had platelet engraftment. Median day of neutrophil and platelet engraftment in group- 1 were day +14 and day +20 while in group- 2 were day +14 and day +19 respectively ( $\mathrm{p}$ value $0.24 \& 0.08$ ). 2 patients who did not have neutrophil engraftment died before that due to MDR sepsis.

Infectious complications:

Proven bacterial infection was seen in $34 \%$ cases in group- 1 and $15 \%$ cases in group- 2 but difference could not reach statistical significance (p value 0.07 ). CMV reactivation was seen in 51\% cases of group- 1 and $65 \%$ patients in group-2 and median duration of reactivation was day +30 in both ( $\mathrm{p}$ value 0.2 ). CMV pneumonitis was seen in 1 patient from each group. Other viral infections including HSV, adeno virus and BK viremia was seen in 5 and 6 patients in group-1 and 2, respectively. Six patients developed invasive fungal infections in study cohort including 3 from each group ( $\mathrm{p}$ value 0.62 ).

Non-infectious complications:

19 patients $(40.4 \%)$ in group-1 and $13(40.6 \%)$ in group-2 developed acute GVHD. 18 patients had grade III-IV GVHD including 9 patients from each group (p value 0.32 ). Total 6 patients (7.5\%) developed chronic GVHD in which 4 from group-1 and 2 from group-2 (p value 1). 28 patients (35\%) developed VOD including 21 patients (44.6\%) in group-1 and $7(21.8 \%)$ in group-2, out of which 24 had mild to moderate grade of VOD. VOD was seen in higher number of cases of group-1 and the difference was significant ( $\mathrm{p}$ value 0.039). 


\section{Chimerism:}

Median chimerism at day +30 and day +90 in group- 1 were $96 \%$ and $97 \%$ while in group- $296 \%$ and $95.5 \%$ respectively. Mixed chimerism was seen in $19(24 \%)$ patients. 3 patients had secondary graft failure and 2 of them underwent re-transplantation and are currently thalassemia free.

\section{Survival}

Overall survival and thalassemia free survival over a median duration of 29 months in whole cohort were $83.5 \%$ and $81 \%$ respectively. Overall survival and thalassemia free survival at 1 year in group-1 were $82.9 \%$ and $80.8 \%$ while in group- $284.3 \%$ and $78.1 \%$ respectively and difference was not statistically significant (p value $0.86 \& 0.76$ ) (figure 1). Most common cause of death was gram negative sepsis followed by severe VOD and grade III-IV GVHD.

\section{B: Aplastic anemia}

Transplant characteristics of 21 patients with SAA (9 in group-1 and 12 in group-2) are mentioned in Table 2 .

Engraftment:

Median day of neutrophil and platelet engraftment in group- 1 were day +13 and day +14 while in group- 2 were day +15 and day +22 respectively ( $\mathrm{p}$ value 0.75 and 0.68 ). Though both neutrophil and platelet engraftment were earlier in group 1, but difference was not significant.

Infectious complications:

Almost all (20 patients) developed fever in neutropenic period but proven bacterial infection was seen in $22.2 \%$ cases in group- 1 and in 50\% cases in group-2 (p value 0.21 ). Total 12 patients had CMV reactivation including 6 patients from each group ( $\mathrm{p}$ value 0.46). 2 patients in group-1 and 1 from group-1 developed invasive fungal disease ( $\mathrm{p}$ value 0.38 ).

Non-infectious complications:

$22.2 \%$ in group- 1 and $25 \%$ patients in group-2 had acute GVHD (p value 0.88 ) but incidence of grade III-IV GVHD was more in group-2, in which all 3 had higher grades of GVHD (p value 0.19). Chronic GVHD incidence in whole cohort was $9.5 \%$ and both patients were from group-2 (p value 0.21 ).

Chimerism:

Median chimerism at day +30 and day +90 were $90 \%$ and $93 \%$ in group- 1 while it was $93 \%$ in group2 (p value 0.91 ) at both time intervals.

Survival:

Overall survival over a median period of follow up of 26 months in group- 1 was $77.8 \%$ OS and $66.7 \%$ in group-2 but difference was not significant statistically ( $\mathrm{p}$ value 0.616 ) (figure 2). Most common cause of death was gram negative sepsis.

\section{C: Immunodeficiency disorders}

Total 10 patients of various immunodeficiency disorders including 3 of primary HLH, 4 congenital neutropenia, 1 Griscelli syndrome, 1 LAD-1 and 1 patient of CGD underwent HSCT. 3 patients were in group-1 and 7 in group-2. Transplant characteristics as shown in table 3

4 patients developed proven bacterial sepsis and CMV disease (colitis) was seen in 1(10\%). None of the patient suffered from fungal infection. Acute GVHD was seen in 20\% cases and chronic GVHD in none. Overall survival and EFS were $70 \%$ and $60 \%$ in the whole cohort and all 3 patients who died were from group 2 , but statistical significance could not be ascertained due to very a smaller number of study subjects (figure 
3). 1 patient of congenital neutropenia had primary engraftment failure but could survive due aggressive support with granulocyte and GCSF in immediate transplant period and will undergo re-transplantation.

\section{D: Hematological malignancy}

Total 7 patients of various hematological malignancy (3 relapsed ALL, 2 AML-CR2, 1 AML in CR-1 and 1 case of JMML) underwent transplant. TBI/Etoposide or Busulphan/Mel with ATG was used for conditioning. Out of 7 patients 5 received horse ATG and 2 rabbit ATG. All patients had neutrophil and platelet engraftment and median chimerism at day +30 and day +90 was $99 \%$ and $100 \%$ respectively. 4 patients developed proven bacterial sepsis and 6 had CMV reactivation. Acute GVHD was seen in $71 \%$ cases. 4 patients had grade I-II GVHD and responded well to prednisolone while 1 had grade III and was a prednisolone poor responder. Overall survival at 6 months was $71.4 \%$ another patient relapsed after 8 months of transplant and died.1 patient relapsed within 6 months and died while another death due to multi-organ toxicities. Another study by Yunsuk Choi has also demonstrated similar survival rate $44.5 \%$ over 3 years. ${ }^{50}$

\section{Discussion}

Allogeneic HSCT has been used to treat hemoglobinopathies, aplastic anemia, immunodeficiency disorders and as salvage therapy for acute leukemia. Despite improvements in HLA matching, quality control measures, and supportive care used in hematopoietic stem cell transplantation, graft-versus-host disease (GVHD) remains a common cause of morbidity and mortality in transplant recipients especially mismatched and unrelated transplants.$^{10,19}$

In various comparison studies, Rabbit ATG more efficiently depletes lymphocytes in vivo and was more cytotoxic on a weight basis in vitro and induces regulatory $\mathrm{T}$ cell development from normal $\mathrm{T}$ cells in tissue culture, which should be beneficial in suppressing a harmful immune mediated or autoimmune pathophysiology \& preserve graft versus leukemia effect. ${ }^{20,21}$ On the other hand, ATG is a differentiation- inducing agent; horse ATG induces growth and differentiation of normal bone marrow and terminal differentiation of the HL 60 cell line (AML-M3). ${ }^{22-24}$ The bone marrow granulocyte colony-forming units increase after ATG treatment. ${ }^{25}$ By adding ATG to the conditioning regimens, the incidence of severe acute and extensive chronic GVHD is less; it is regarded as a better conditioning therapy for HLA-mismatched transplants. However, the comparative efficacy rabbit ATG and horse ATG in pre-HSCT conditioning has not been studied. We did this retrospective analysis to look for outcomes in relation to type of ATG used.

It was speculated initially that rabbit ATG use will reduce incidence of graft rejection and acute and chronic GVHD on the cost of increased infectious complications due to prolonged lymphotoxic effect but on contrary being a steroid sparing agent by less GVHD occurrence and with better supportive care and careful vigilance which has evolved over a period of time might give better outcome.

In our study of thalassemia patients, group 1 patients had more bacterial infections but that result seems to be biased as in group 1 patients were older than group 2 and were of class -III with iron overload hence risk of veno-occlusive disease, delayed engraftment and infection was anyway higher but overall incidence reported (13-25\%) was similar to other studies. ${ }^{26-27}$

Fungal infection risk was similar to other studies which reported $4-10 \%$ risk. Though viral infections especially CMV reactivation rate was higher in group 2, but difference was not significant and CMV disease was seen in only $2.5 \%$ cases and was same in both groups.

Acute GVHD risk was approximately $40 \%$ in whole study cohort which is akin to results from Bacigalupo et al who has reported GVHD incidence of $11-41 \%$ and $39 \%$ risk of chronic GVHD which is quite higher then seen in our center. In this comparison was made between group with or with ATG and low $(7.5 \mathrm{mg} / \mathrm{kg})$ and high ATG $(15 \mathrm{mg} / \mathrm{kg})$ dose and was seen that high dose ATG was significantly associated with increased risk of infection but reduction in GVHD incidence was seen with high ATG dose which is quite similar to our study in which low dose ATG was used and associated with only $7.5 \%$ incidence of chronic GHVD and only 2 patients had extensive GVHD.${ }^{10} \mathrm{~A}$ study by Zakerinia et al on Iranian population in which horse ATG was used showed 15-17\% risk of acute GVHD in a comparison to no ATG group (27\% risk), 40mg/kg and 
$100 \mathrm{mg} / \mathrm{kg}$ doses were used respectively. Other study in adult population showed similar incidence of acute and chronic GVHD in horse and rabbit ATG group. ${ }^{10,25,28}$

Veno-occlusive disease was seen in $35 \%$ of whole cohort and was higher in group- 1 than group- 2 and difference was significant ( $\mathrm{p}$ value 0.039 ) which can be explained by relatedly older age and class III thalassemia were there in group-1.

Median chimerism in two groups were similar at 1 and 3 months in post-transplant period. $24 \%$ patients had mixed chimerism and $3(3.7 \%)$ patients had graft rejection which is comparable to $4-13 \%$ risk found in other studies done by Zakerinia et al, Li et al, and Lawson et al. ${ }^{29,}{ }^{30}$ Horse and rabbit ATG were used respectively in these different study populations.

Overall survival and thalassemia free survival were $83.5 \%$ and $81 \%$ successively and difference between two study group was also similar (p value $0.86 \& 0.76$ ). OS and TFS was quite less before ATG era but has improved significantly from $80-100 \%$ in various studies using different ATG preparations with Bu-Cy-ATG based conditioning and In a recent study by Bernardo et al. showed that the use of treosulfan-based regimens in 20 thalassemic patients resulted in a probability of OS and TFS of $95 \%$ and $85 \%$, respectively. ${ }^{29-31}$

In Severe aplastic anemia both immunosuppression and HSCT has been used for management of SAA but studies shows benefit of using HSCT as primary modality due to poor complete response rate of 50-60\% with immunosuppression and 8-13\% risk of clonal evolution over a period of time. ${ }^{32-35}$

Though studies have shown the addition of ATG in conditioning has reduced graft rejection and GVHD incidence but whether source of ATG affect outcome was not analyzed earlier. Horse ATG was used in a dose of $90 \mathrm{mg} / \mathrm{kg}$ and rabbit ATG in $3 \mathrm{mg} / \mathrm{kg} /$ day with flu-cy as standard conditioning regimen. ${ }^{36}$

In our study group-1 had early neutrophil and platelet engraftment and less incidence of bacterial infection , which correlated with early neutrophil engraftment but due to less number of study subjects statistical significance couldn't be ascertained (p value 0.75 and 0.68 ) which is similar to Atta et al who have found higher incidence of bacterial and fungal infections in rabbit ATG group done but in contrast to study done by Kekre et al who didn't found any difference in infectious complications. ${ }^{37-39}$

Acute GVHD incidence in group- 1 and 2 were $22.5 \%$ and $25 \%$ respectively which is similar to other studies that have shown a $11 \%-40 \%$ risk associated but we found a higher occurrence of GVHD in group- 2 though not significant ( $\mathrm{p}$ value 0.88 ) which is in contrast to studies by Kekre et al who analyzed 546 SAA transplants between 2008 and 2013 using MSD BM transplantation. aGVHD $(17 \%$ versus $6 \%, \mathrm{P}<.001)$ that can be explained by all 3 patients who received haplo-identical transplant were in group- 2 and PBSC was more commonly used as stem cell source for expected early engraftment. ${ }^{37}$

Chronic GVHD incidence in our study was $9.5 \%$ and was similar in both groups ( $\mathrm{p}$ value 0.21 ) compared to other studies might be related to common use of PBSC as stem cell source but other studies favor use of rabbit ATG over horse ATG as first one is associated with less incidence of chronic GVHD. ${ }^{37-39}$

We found a high overall survival in group- 1 of $77.8 \%$ than in group- 2 of $67 \%$ but difference was not statistically significant ( $\mathrm{p}$ value 0.616). Higher survival in group-1 was not as expected but as other results as early neutrophil engraftment, lesser bacterial infections and slightly lower GVHD incidence might have resulted in improved survival. Probable explanation of better outcome in rabbit ATG group in earlier comparative studies was expansion of Tregs by rabbit ATG only, which is proven in both in vivo and in vitro studies but this effect was probably negated by more potent suppression of CD4 cells which have other positive effects on hematopoiesis and in addition, horse serum might contribute to recovery of hematopoiesis by stimulatory effects in the bone marrow. ${ }^{37-38,40,41}$ More prolonged lymphopenia after rabbit ATG might impair marrow recovery as stimulatory cytokines derived from T-cells are depleted. ${ }^{42}$

In Primary immunodeficiency disorders (PID) group, haplo-identical transplant was used in 5 patients due to an urgent need of transplant, as most of the patients were referred either in accelerated phase of disease in HLH or with active infections in others. There were differences in two groups for various transplant 
characteristics, CD34 dose, conditioning regimen and GVHD prophylaxis as 4 patients of haplo-identical transplants received rabbit ATG. Relative higher mortality is well explained by similar fact, but no statistical difference was found when compared in two ATG groups due to very a smaller number of study subjects. Graft rejection was seen in $10 \%$ of cases. Overall survival and event free survival in our study were $70 \%$ and $60 \%$ which is similar to other reported studies with $60-90 \%$ OS. ${ }^{43,44}$

\section{Limitation:}

As it was a retrospective analysis and a small number of study patients were included in study especially in PID and hematological malignancy group so further prospective randomized studies are required to confirm our study results.

\section{Conclusion:}

Effectiveness of rabbit and horse ATG were similar in thalassemia patients while in SAA equine ATG use was associated with early neutrophil engraftment and less incidence of bacterial infection but OS in both groups were comparable. In primary immunodeficiency disorders also, equine ATG use was associated better overall survival but statistical significance could not be ascertained due to very less number of study subjects.

Though ATG is a well-known biological compound for decades, several aspects about its mode of action are yet to be unveiled. Immunological interaction of ATG are quite complex in vivo and resultant outcome depends upon which action predominates. Careful selection of pre-transplant conditioning can significantly reduce transplant related mortality and increase the probability of durable engraftment. Horse or rabbit ATG can be used in pre-stem cell transplant conditioning depending upon availability and ease of administration.

\section{Conflict of Interest Statement: None of the authors have any conflict of interest to declare.}

\section{References}

1. Kröger N, Solano C, Wolschke C, Bandini G, PatriarcaF,Pini M, Nagler A, Selleri C, Risitano A, Messina G, Bethge W, Pérez de Oteiza J, Duarte R, et al. Antilymphocyte Globulin for Prevention of Chronic Graft-versus-Host Disease. N Engl J Med. 2016; 374(1): 43-53.

2. Walker I, Panzarella T, Couban S, Couture F, Devins G, Elemary M, Gallagher G, Kerr H, Kuruvilla J, Lee SJ, Moore J, Nevill T, Popradi G, et al. Pretreatment with antithymocyte globulin versus no anti-thymocyte globulin inpatients with haematological malignancies undergoing haemopoietic cell transplantation from unrelated donors: a randomised, controlled, open-label, phase 3, multicenter trial. Lancet Oncol. 2016; 17(2): 164-173.

3. Yu ZP, Ding JH, Wu F, Liu J, Wang J, Cheng J, Zhao G. Quality of life of patients after allogeneic hematopoietic stem cell transplantation with antihuman thymocyte globulin. Biol Blood Marrow Transplant. 2012; 18(4): 593-599.

4. M. Zakerinia, H.N. Khojasteh.Bone Marrow Transplantation in Thalassemia Major Patients Using "Short" Anti-Thymocyte Globulin Therapy in Shiraz, Southern Iran.Transplantation Proceedings. 2005; 37: 44774481.

5. Brennan DC, Flavin K, Lowell JA, et al. A randomized, double-blinded comparison of Thymoglobulin versus Atgam for induction immunosuppressive therapy in adult renal transplant recipients. Transplantation. 1999; 67:1011-1018. [Erratum, Transplantation 1999;67:1386.]

6. Gaber AO, First MR, Tesi RJ, et al. Results of the double-blind, randomized, multicenter, phase III clinical trial of Thymoglobulin versus Atgam in the treatment of acute graft rejection episodes after renal transplantation. Transplantation. 1998;66:29-37.

7. Di Bona E, Rodeghiero F, Bruno B, et al. Rabbit antithymocyte globulin (r-ATG) plus cyclosporine and granulocyte colony stimulating factor is an effective treatment for Aplastic Anaemia Patients Unresponsive 
to a First Course of Intensive Immunosuppressive Therapy. Gruppo Italiano Trapianto Di Midollo Osseo (GITMO). Br J Haematol. 1999 Nov;107(2):330-334.

8. Scheinberg P, Nunez O, Young NS. Retreatment with rabbit anti-thymocyte globulin and ciclosporin for patients with relapsed or refractory severe aplastic anaemia. Br J Haematol. 2006;133:622-627.

9. Scheinberg P, Fischer SH, Li L, et al. Distinct EBV and CMV reactivation patterns following antibodybased immunosuppressive regimens in patients with severeaplastic anemia. Blood. 2007;109:3219-3224.

10. Bacigalupo A, Lamparelli T, Bruzzi P, et al. Antithymocyte globulin for graft-versus-host disease prophylaxis in transplants from unrelated donors: 2 randomized studies from Gruppo ItalianoTrapiantiMidollo Osseo (GITMO). Blood. 2001;98:2942-2947.

11.Remberger M, Sundberg B. Rabbit-immunoglobulin G levels in patients receiving thymoglobulin as part of conditioning before unrelated donor stem cell transplantation. Haematologica. 2005;90:931-938.

12. Kim HJ, Min WS, Cho BS, et al. Successful prevention of acute graftversus- host disease using lowdose antithymocyte globulin after mismatched, unrelated, hematopoietic stem cell transplantation for acute myelogenous leukemia. Biol Blood Marrow Transplant. 2009;15: 704-717.

13. Bacigalupo A, Lamparelli T, Barisione G, et al. Thymoglobulin prevents chronic graft-versus-host disease, chronic lung dysfunction, and late transplant-related mortality: long-term follow-up of a randomized trial in patients undergoing unrelated donor transplantation. Biol Blood Marrow Transplant. 2006;12:560-565.

14. Bacigalupo A, Lamparelli T, Milone G, et al. Pre-emptive treatment of acute GVHD: a randomized multicenter trial of rabbit anti-thymocyte globulin, given on day 7 after alternative donor transplants. Bone Marrow Transplant 2010;45:385-391.

15. Russell JA, Turner AR, Larratt L, et al. Adult recipients of matched related donor blood cell transplants given myeloablative regimens including pretransplant antithymocyteglobulin have lower mortality related to graft-versus-host disease: a matched pair analysis. Biol Blood Marrow Transplant. 2007;13:299-306.

16. Bredeson CN, Zhang MJ, Agovi MA, et al. Outcomes following HSCT using fludarabine, busulfan, and thymoglobulin: a matched comparison to allogeneic transplants conditioned with busulfan and cyclophosphamide. Biol Blood Marrow Transplant. 2008;14:993-1003.

17. Finke J, Bethge WA, Schmoor C, et al. Standard graft-versus-host disease prophylaxis with or without antieT-cell globulin in haematopoietic cell transplantation from matched unrelated donors: a randomised, open-label, multicentre phase 3 trial. Lancet Oncol. 2009;10: 855-864.

18. Yunsuk Choi, Ho Sup Lee et al.The Impact of Antithymocyte Globulin (ATG) Dose in Allogeneic Hematopoietic Stem Cell Transplantation with HLA Mismatched Donors.Blood 2016 128:3393

19. Bacigalupo A, Oneto R, Bruno B, et al. Current results of bone marrow transplantation in patients with acquired severe aplastic anemia. Report of the European Group for Blood and Marrow Transplantation. On behalf of the Working Party on Severe Aplastic Anemia of the European Group for Blood and Marrow Transplantation. Acta Haematol. 2000;103(1):19-25.

20. Feng X, Kajigaya S, Solomou EE, et al. Rabbit ATG but not horse ATG promotes expansion of functional CD4+CD25high FOXP3+ regulatory T cells in vitro. Blood. 2008;111:3675-3683.

21. Lopez M, Clarkson MR, Albin M, Sayegh MH, Najafian N. A novel mechanism of action for antithymocyte globulin:induction of CD4+CD25+Foxp3+ regulatory T cells. J Am Soc Nephrol 2006;17: 28442853.

22. Hunter RF, Mold NG, Brain MR, et al: Differentiation of normal marrow and HL 60 cells induced by antithymocyte globulin. Proc NatiAcad Sci U S A. 1985; 82:4823. 
23. Plantarias L, Gascon P, Bielory L, et al: Lymphocyte phenotype and lymphokines following antithymocyte globulin therapy in patients with aplastic anemia. Br J Hematol,1987; 66:437.

24. Faillc A, Barrctt AJ, Balttrand N, et al: Effect of antilymphocyte globulin on granulocyte precursors in aplastic anemia. Br J Heamatol. 1979; 42:341.

25. Zakerinia M, NouraniKhojasteh H, Ramzi M, et al: Bone marrow transplantation for thalassemia in Shiraz. Transplant Proc. 1995; 27:2659.

26.M Mohty1,2, W Jacot et al.Infectious complications following allogeneic HLA-identical sibling transplantation with antithymocyte globulin-based reduced intensity preparative regimen. Leukemia. 2003; 17: $2168-2177$.

27. E Goussetis1, I Peristeri1 et al. HLA-matched sibling stem cell transplantation in children with bthalassemia with anti-thymocyte globulin as part of the preparative regimen: the Greek experience. Bone Marrow Transplantation. 2012; 47: 1061-1066

28.Crystal Lu ,KlodianaNemeet al.Horsies and Bunnies: A Comparison of Antithymocyte Globulin in Allogeneic Stem Cell Transplant. Biol Blood Marrow Transplant. 2018; 24: S119-S290.

29.Li CK, Shing MMK, Chik KW, Lee V, Leung TF, Cheung AYK et al. Haematopoietic stem cell transplantation for thalassaemia major in Hong Kong: prognostic factors and outcome. Bone Marrow Transplant. 2002; 29: $101-105$.

30.Lawson SE, Roberts IAG, Amrolia P, Dokal I, Szydlo R, Darbyshire PJ. Bone marrow transplantation for b-thalassaemia major: the UK experience in two paediatriccentres. Br J Haematol. 2003; 120: 289 - 295.

31.Bernardo ME, Zecca M, Piras E, Vacca A, Giorgiani G, Cugno C et al. Treosulfan based conditioning regimen for allogeneic haematopoietic stem cell transplantation in patients with thalassemia major. Br $\mathrm{J}$ Haematol 2008; 143: $548-551$.

32. Mathe G, Amiel JL, Schwarzenberg L, et al. Bone marrow graft in man after conditioning by

Anti lymphocytic serum. Br Med J. 1970; 2:131-136.

33.Socie G, Henry-Amar M, Bacigalupo A, Hows J, Tichelli A, Ljungman P, et al. Malignant tumors occurring after treatment of aplastic anemia. European Bone Marrow Transplantation-Severe Aplastic Anaemia Working Party. N Engl J Med. 1993; 329(16):1152-1157.

34. Ohara A, Kojima S, Hamajima N, Tsuchida M, Imashuku S, Ohta S, et al. Myelodysplastic syndrome and acute myelogenous leukemia as a late clonal complication in children with acquired aplastic anemia. Blood. 1997; 90(3):1009-1013.

35. Tichelli A, Gratwohl A, Wursch A, Nissen C, Speck B. Late haematological complications in severe aplastic anaemia. Br J Haematol. 1988; 69(3):413-418.

36. Georges and Rainer Storb Hematopoietic stem cell transplantation for acquired aplastic anemia George E. 1,21Clinical Research Division, Fred Hutchinson Cancer Research Center, University of Washington, Seattle, WA2Department of Medicine, University of Washington, Seattle, WA; Curr Opin Hematol. 2016 November; 23(6): 495-500

37.Natasha Kekre, Ying Zhang, Mei-Jie Zhang, Jeanette Carreras et al: Effect of antithymocyte globulin source on outcomes of bone marrow transplantation for severe aplastic anemia Haematologica2017 Volume 102(7):1291-1298.

38. Atta EH, de Sousa AM, Schirmer MR, Bouzas LF, Nucci M, Abdelhay E. Different outcomes between cyclophosphamide plus horse or rabbit antithymocyte globulin for HLA-identical sibling bone marrow transplant in severe aplastic anemia. Biol Blood Marrow Transplant. 2012; 18:1876-1882. 
39. Xin Chen, JialinWei, Yong Huang et al; Effect of Antithymocyte Globulin Source on Outcomes of HLAMatched Sibling Allogeneic Hematopoietic Stem Cell Transplantation for Patients with Severe Aplastic Anemia. Biol. Bone Marrow Transplant. 2018;24:86-90.

40. Kawano Y, Nissen C, Gratwohl A, Wursch A, Speck B. Cytotoxic and stimulatory effects of antilymphocyte globulin (ALG) on hematopoiesis. Blut. 1990; 60:297-300.

41. Barbano G, Schenone A, Roncella S, et al. Anti-lymphocyte globulin stimulates normal human T cells to proliferate and to release lymphokines in vitro. A study at the clonal level. Blood. 1988;72:956-963.

42. Naparstek E, Delukina M, Or R, et al. Engraftment of marrow allografts treated with Campath1monoclonal antibodies. Experimental Hematology. 1999; 27:1210-1218.

43.Grunebaum E, MazzolariE et al. Bone marrow transplantation for severe combined immune deficiency. JAMA 2006;295:508-518.

44. Hossam K. Mahmoud et al. Allogeneic hematopoietic stem cell transplantationfor non-malignant hematological disorders. Journal of Advanced Research. 2015; 6: 449-458.

45.Hematopoietic Stem Cell Transplantation for Primary Immunodeficiencies By Elizabeth Kang and Andrew Gennery. Hematol Oncol Clin North Am. 2014 December ; 28(6): 1157-1170.

\section{LEGENDS}

\section{Tables}

Table 1: Explains the transplant characteristics of Thalassemia patients with regards to demographic characteristics, type of transplant and conditioning in the two study groups.

Table 2: Explains the transplant characteristics of severe aplastic anemia patients with regards to demographic characteristics, type of transplant and conditioning in the two study groups.

Table 3: Explains the transplant characteristics of primary immunodeficiency disorder patients with regards to demographic characteristics, type of transplant and conditioning in the two study groups. The outcomes in this patient population with regards to the two groups is also explained.

\section{Figures}

Figure 1: Kaplan-Meier survival curve for Thalassemia patients in the two study groups

Figure 2: Kaplan-Meier survival curve for severe aplastic anemia patients in the two study groups

Figure 3: Kaplan-Meier survival curve for primary immunodeficiency disorder patients in the two study groups

\section{Hosted file}

Table 1.docx available at https://authorea.com/users/347735/articles/473303-comparisonbetween-equine-and-rabbit-anti-thymocyte-globulin-atg-used-in-conditioning-forpediatric-patients-undergoing-hematopoietic-stem-cell-transplantation

\section{Hosted file}

Table 2 .docx available at https://authorea.com/users/347735/articles/473303-comparisonbetween-equine-and-rabbit-anti-thymocyte-globulin-atg-used-in-conditioning-forpediatric-patients-undergoing-hematopoietic-stem-cell-transplantation

\section{Hosted file}

Table 3 .docx available at https://authorea.com/users/347735/articles/473303-comparisonbetween-equine-and-rabbit-anti-thymocyte-globulin-atg-used-in-conditioning-forpediatric-patients-undergoing-hematopoietic-stem-cell-transplantation 
Survival Functions

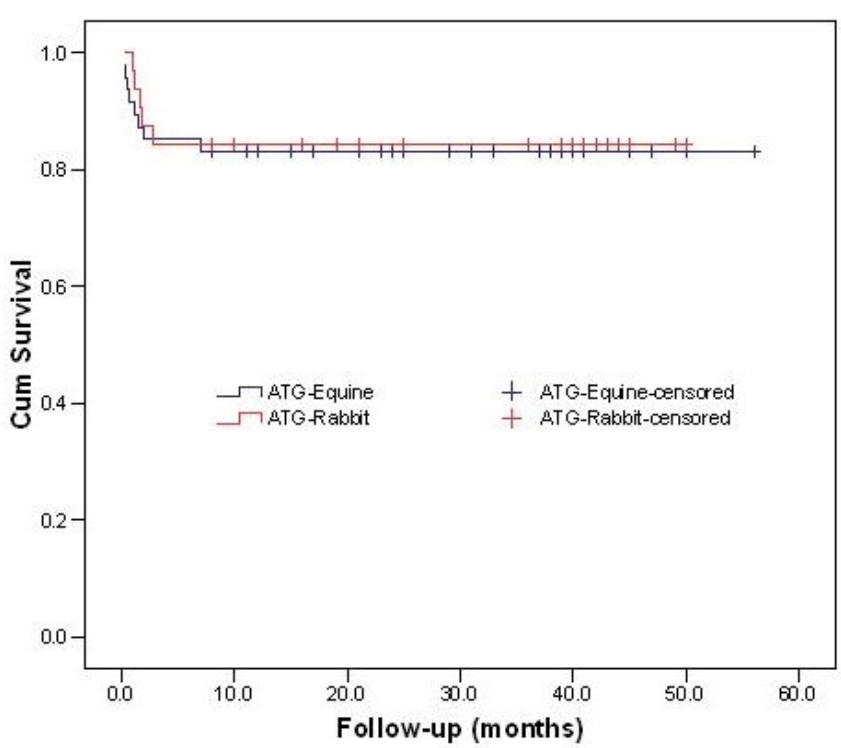

Survival Functions

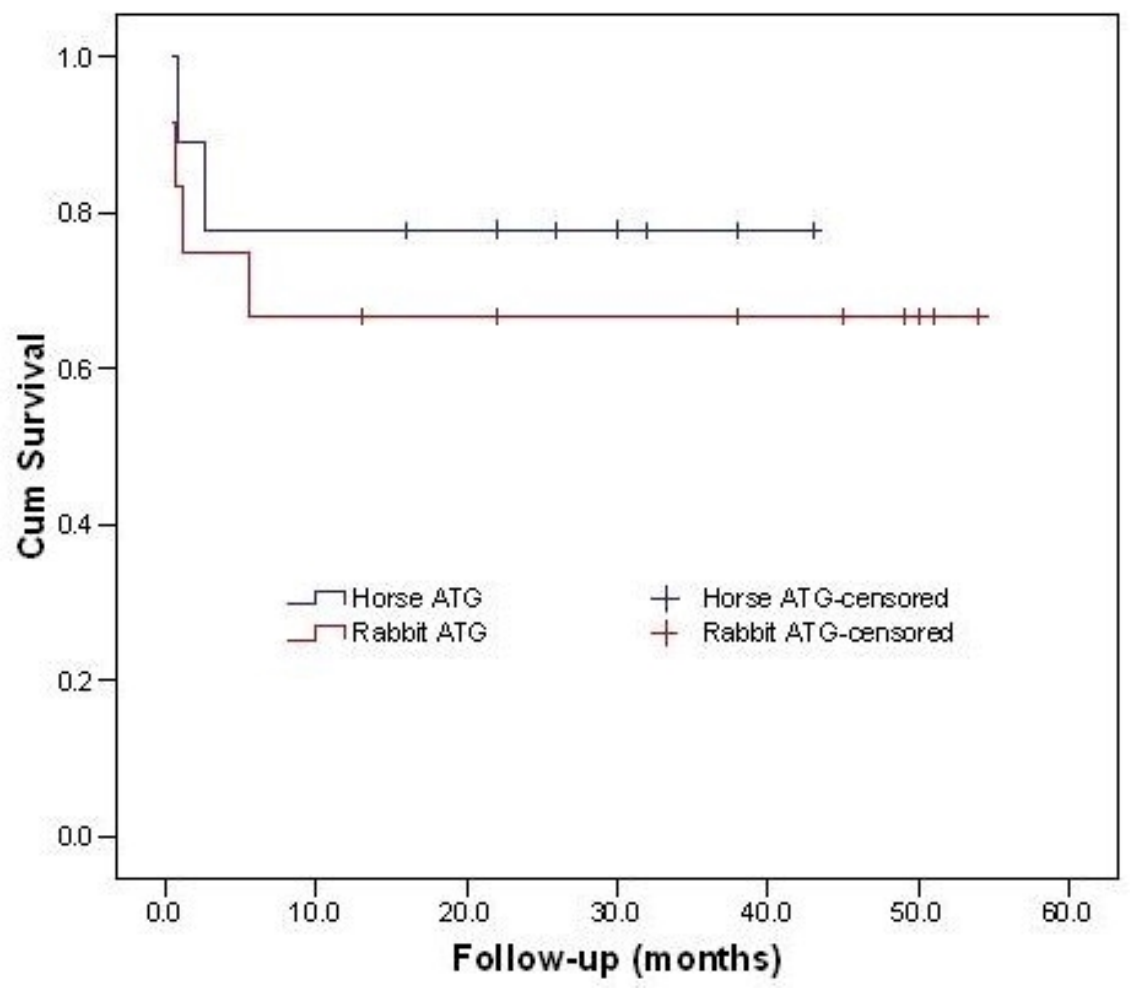


Survival Functions

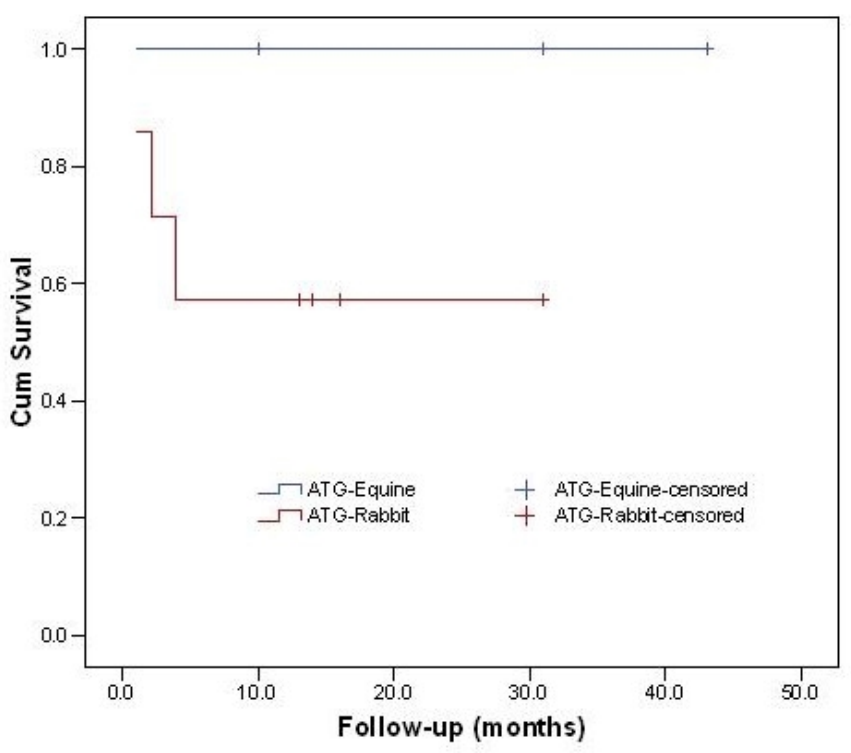

